Supporting Information

\title{
Effects of Low-Molecular-Weight Organics on the Photoreduction of Bromate in Water
}

\author{
Zhihao Chen, ${ }^{1,2}$ Jiyuan Jin, ${ }^{1}$ Xiaojie Song, ${ }^{1}$ Shijie Wei, ${ }^{1}$ Li Zhang, ${ }^{1}$ Shujuan Zhang ${ }^{1, *}$
}

${ }^{1}$ State Key Laboratory of Pollution Control and Resource Reuse, School of the Environment, Nanjing University, 163 Xianlin Avenue, Nanjing, 210023, China

${ }^{2}$ NUS Environmental Research Institute, National University of Singapore, 5A Engineering Drive 1, \#02-01, Singapore 117411, Singapore

*Correspondence author. Phone: +86 258968 0389, E-mail: sjzhang@nju.edu.cn

Submitted to: ACS ES\&T Engineering

This file contains 5 figures, 4 Tables and 19 references. 

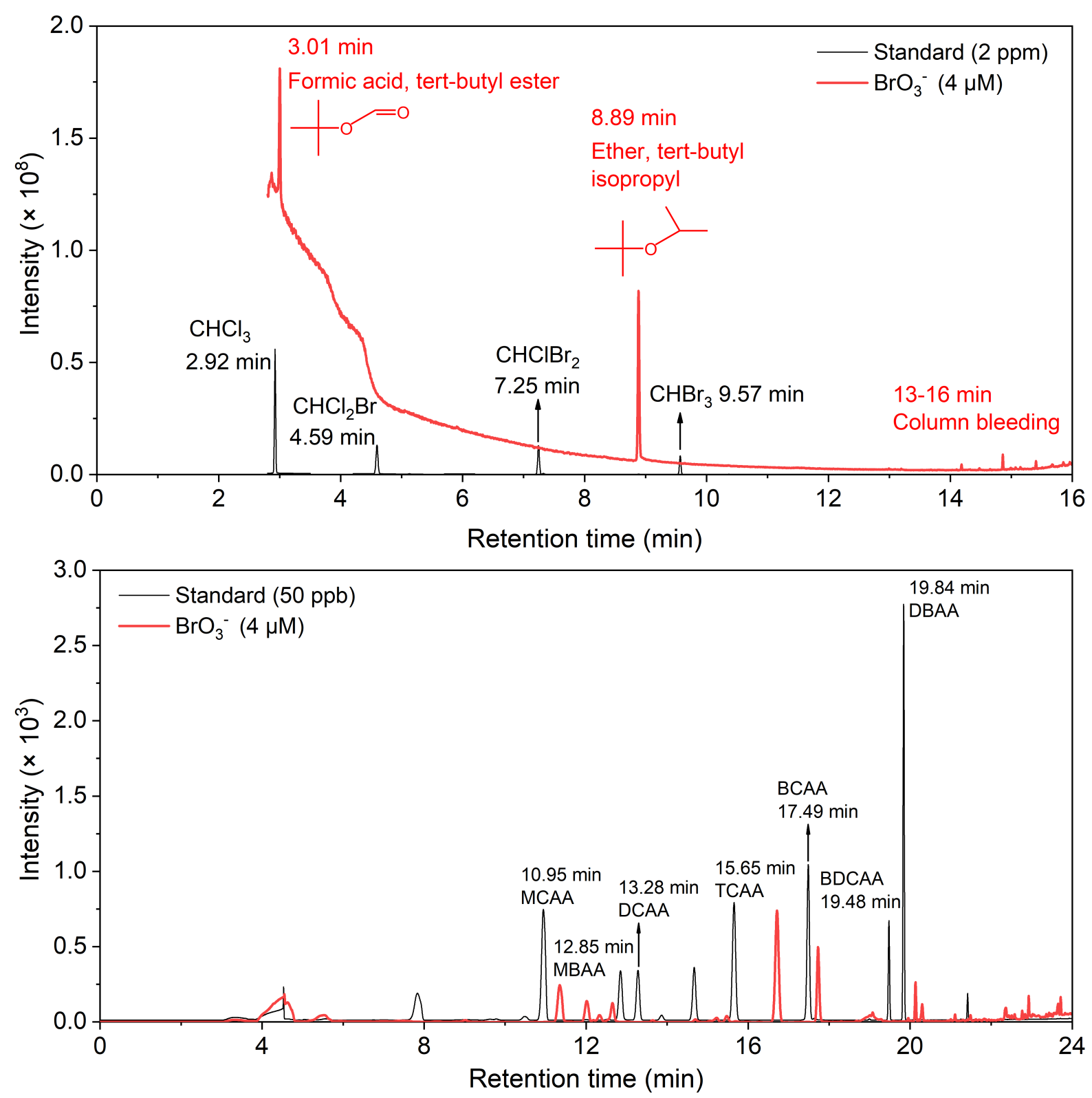

Figure S1. Detection of organic halogens in bromate reduction by UV/AA. AA: $10 \mu \mathrm{M}, \mathrm{BrO}_{3}{ }^{-}: 4$ $\mu \mathrm{M}$, light intensity: $8.59 \mathrm{~mW} \cdot \mathrm{cm}^{-2}$. The peaks after $13 \mathrm{~min}$ in the top panel are siloxane-based compounds due to the column bleeding. (Top) With four common THMs as standards, (Bottom) With eight common HAAs as standards. 


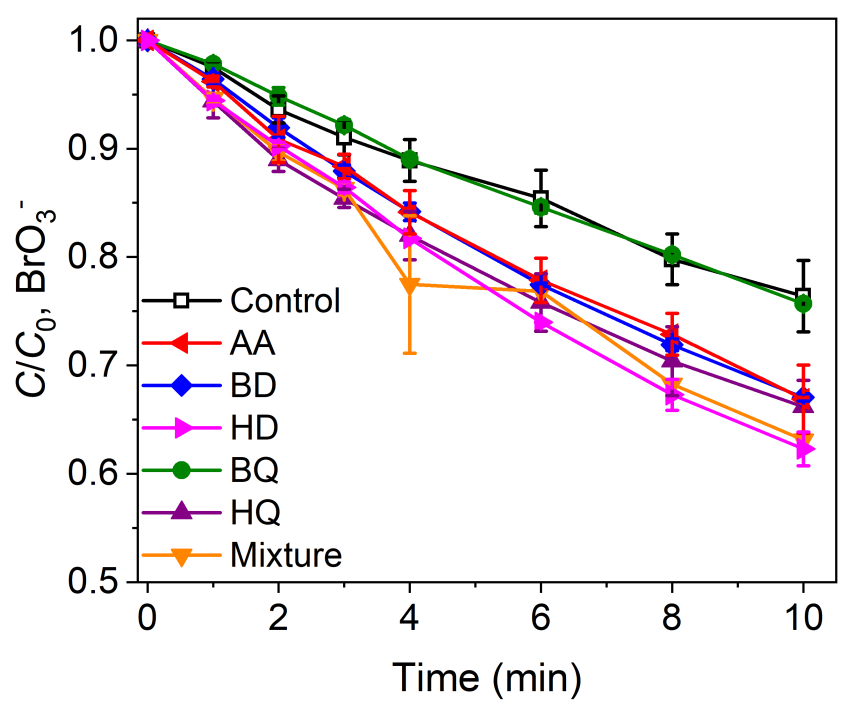

Figure S2. The $\mathrm{BrO}_{3}{ }^{-}$reduction by $\mathrm{LMWO}$ under UV irradiation. $\left[\mathrm{BrO}_{3}{ }^{-}\right]=4 \mu \mathrm{M},[\mathrm{AA}]=[\mathrm{BD}]$

$=[\mathrm{HD}]=[\mathrm{BQ}]=[\mathrm{HQ}]=50 \mu \mathrm{M}$, mixture: a mixed solution of FoA, AcA, PyA, OxA, FoD, TBA, IPA, Ace, AcD and $\mathrm{LaA}$ at a concentration $5 \mu \mathrm{M}$ for each, light intensity: $6.9 \mathrm{~mW} \cdot \mathrm{cm}^{-2}$.

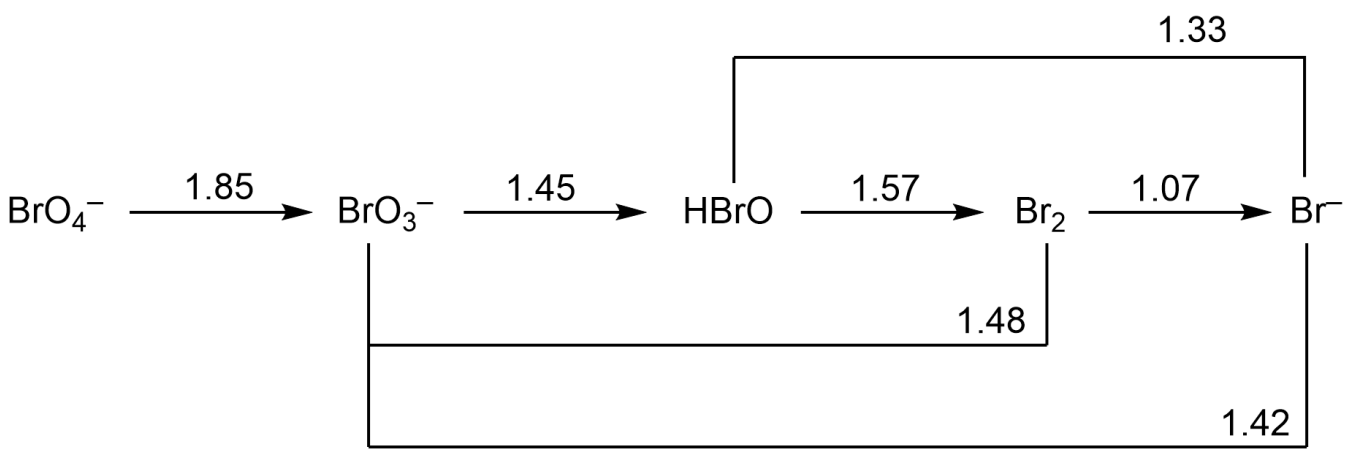

Figure S3. Standard redox potentials ( $E^{0}$ in V vs SHE) of bromine species. ${ }^{1}$ 


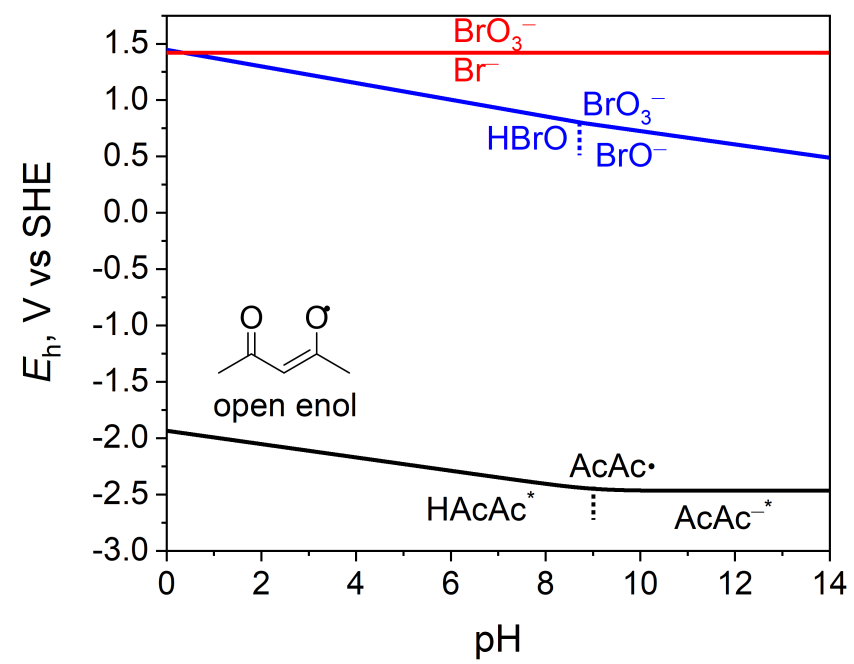

Figure S4. $E_{\mathrm{h}}-\mathrm{pH}$ diagrams of bromine species and excited AA (HAcAc). ${ }^{1,2}$

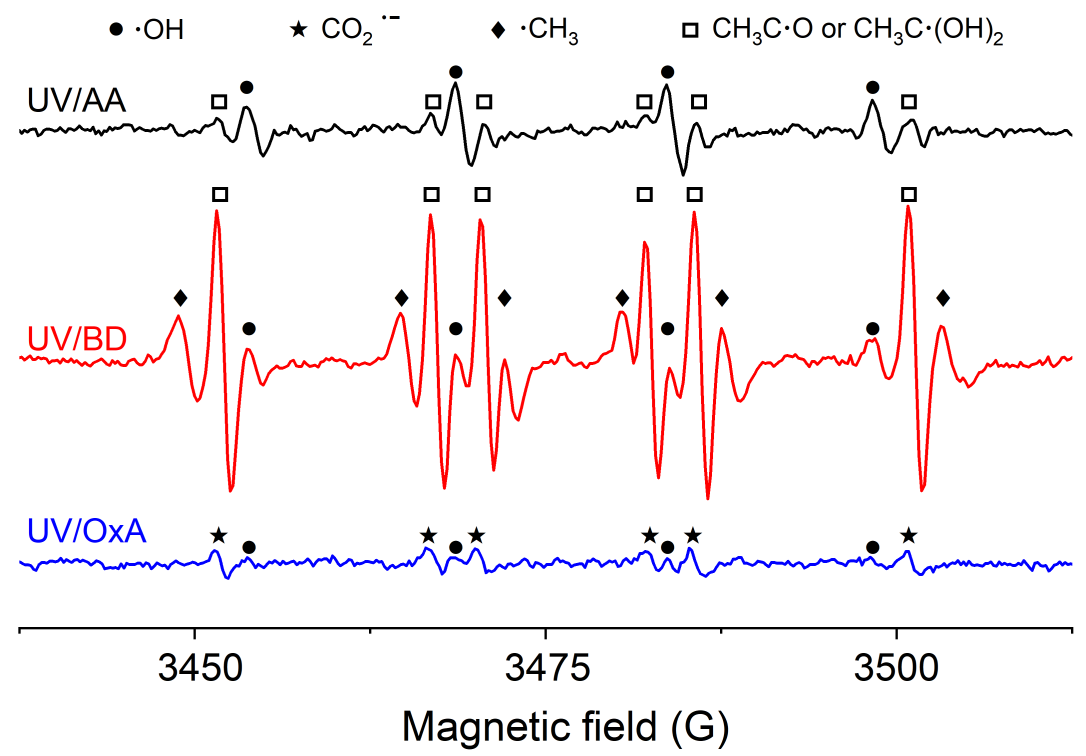

Figure S5. ESR spectra of UV, UV/BD, UV/OxA and UV/AA processes after irradiation using DMPO as quencher. Irradiance time for UV/AA and UV/BD is 2 min while $3 \mathrm{~min}$ for UV and $\mathrm{UV} / \mathrm{OxA}$ process. $[\mathrm{BD}]=[\mathrm{AA}]=1.0 \mathrm{mM},[\mathrm{OxA}]=5 \mathrm{mM},[\mathrm{DMPO}]=100 \mathrm{mg} \cdot \mathrm{L}^{-1}$, light intensity: $0.85 \mathrm{~mW} \cdot \mathrm{cm}^{-2}$. For $\cdot \mathrm{OH}: \mathrm{A}_{\mathrm{N}}=14.9 \mathrm{G}, \mathrm{A}_{\mathrm{H}}=14.9 \mathrm{G}$; for $\mathrm{CO}_{2} \cdot \mathrm{A}_{\mathrm{N}}=15.5 \mathrm{G}, \mathrm{A}_{\mathrm{H}}=3.1 \mathrm{G}$, consistent with reported values. ${ }^{3}$ Radicals of $\cdot \mathrm{CH}_{3}$ and $\mathrm{CH}_{3} \mathrm{C} \cdot \mathrm{O}$ or $\mathrm{CH}_{3} \mathrm{C} \cdot(\mathrm{OH})_{2}$ are identified form previous research. $^{4}$ 
Table S1. Reported concentrations of bromate in variant water sources.

\begin{tabular}{lll}
\hline Water source & Bromate $\left(\mu \mathrm{g} \cdot \mathrm{L}^{-1}\right)$ & Generation pathways \\
\hline Hydraulic fracturing wastewater $(\mathrm{HFW})$ & $33.6-347.6$ & Ozonation $\left(3.3 \mathrm{mg} \cdot \mathrm{L}^{-1}\right)$ of Ohio River water with $0-0.1 \% \mathrm{HFW}$ \\
Wastewater treatment plants in Swiss & $<2$ to $>400$ & Ozonation: $0.5,1,1.5 \mathrm{mg} \mathrm{O} / \mathrm{mg}$ DOC \\
An aquifer in the United Kingdom & 2000 & Caused by historical spillage from a chemical plant \\
Natural water & $<2-150$ & Ozonation under normal drinking water treatment conditions \\
\end{tabular}


Table S2. Reported concentrations of low-molecular-weight-organics (LMWOs) in water.

\begin{tabular}{|c|c|c|c|}
\hline Source & Organics & Concentration or formation rate & Ref \\
\hline Soil leachate & $\begin{array}{l}\text { citric, shikimic, oxalic, fumaric, formic, acetic, } \\
\text { malonic, malic, lactic and } t \text {-aconitic acids }\end{array}$ & $1-1100 \mu \mathrm{M}$ & 10 \\
\hline \multirow[t]{2}{*}{ Rain, cloud water, fog water, snow } & formic acid & $0.1-60 \mu \mathrm{M}$ & 11 \\
\hline & acetic acid & $0-296 \mu \mathrm{M}$ & \\
\hline Natural water & natural organic matter & $0-10 \mathrm{mg} \cdot \mathrm{L}^{-1}$ & 12 \\
\hline \multirow[t]{2}{*}{ Seawater, exposed to sunlight } & formaldehyde & $5-72 \mathrm{nM}$ & 13 \\
\hline & acetaldehyde & $2-240 \mathrm{nM}$ & \\
\hline Seawater, exposed to solar radiation & pyruvate & $12.9 \mathrm{nM} /(\mathrm{W} \bullet \mathrm{h}) \mathrm{m}^{-2}$ & 14 \\
\hline Coastal and oceanic surface water & $\begin{array}{l}\text { formaldehyde, acetaldehyde, propanol, glyoxal, } \\
\text { methylglyoxal, glyoxylic acid and pyruvic acid }\end{array}$ & Several $\mathrm{nM} \cdot \mathrm{h}^{-1}$ & 15 \\
\hline Natural water exposed to sunlight & formaldehyde, acetaldehyde, glyoxylate & $1.2-383 \mathrm{nM} /(\mathrm{W} \cdot \mathrm{h}) \mathrm{m}^{-2}$ & 16 \\
\hline Hydraulic fracturing wastewater & isopropanol, acetaldehyde, citric acid & molar per liter level & 17 \\
\hline Three Norwegian waters & molecular weight $<1000$ Dalton & $16-38 \%$ of the total organic carbon & 18 \\
\hline
\end{tabular}


Table S3. The photolysis rate constants of AA at different conditions. [AA]: $0.5 \mathrm{mM}$, $\left[\mathrm{BrO}_{3}^{-}\right]: 0.2 \mathrm{mM}$, light intensity: $4.3 \mathrm{~mW} \cdot \mathrm{cm}^{-2}$.

\begin{tabular}{lllll} 
& \multicolumn{3}{c}{$\boldsymbol{k}_{\mathbf{1}}\left(\mathbf{m i n}^{-1}\right)$} \\
\cline { 2 - 5 } & Control & $\mathrm{O}_{2}$ & $\mathrm{~N}_{2}$ & $\mathrm{~N}_{2} \mathrm{O}$ \\
\hline $\mathrm{W} / \mathrm{O} \mathrm{BrO}_{3}{ }^{-}$ & 0.0425 & 0.0369 & 0.0288 & 0.0337 \\
$\mathrm{~W} / \mathrm{BrO}_{3}{ }^{-}$ & 0.0506 & 0.0460 & 0.0324 & 0.0325 \\
\hline
\end{tabular}


Table S4. The main reactions involved in the photolysis of $\mathrm{BrO}_{3}{ }^{-19}$

Forward reactions that lead to the reduction of $\mathrm{BrO}_{3}{ }^{-}$

$$
\begin{aligned}
& \mathrm{BrO}_{3}{ }^{-}+h v \rightarrow\left(\mathrm{BrO}_{3}^{-}\right)^{*} \rightarrow \mathrm{BrO}_{3} \cdot+\mathrm{e}_{\mathrm{aq}}{ }^{-} \\
& \mathrm{BrO}_{3}{ }^{-}+h v \rightarrow\left(\mathrm{BrO}_{3}^{-}\right)^{*} \rightarrow \mathrm{BrO}_{2} \cdot+\mathrm{O}^{--} \\
& \left.\mathrm{BrO}_{3}{ }^{-}+h v \rightarrow\left(\mathrm{BrO}_{3}{ }^{-}\right)^{*} \rightarrow \mathrm{BrO}_{2}{ }^{-}+\mathrm{O}^{3} \mathrm{P}\right) \\
& \mathrm{BrO}_{3}^{-}+h v+\mathrm{H}^{+} \rightarrow\left(\mathrm{BrO}_{3}^{-}\right)^{*} \rightarrow \mathrm{HBrO}+\mathrm{O}_{2} \\
& \mathrm{BrO}_{3} \cdot+h v \rightarrow \mathrm{BrO} \cdot+\mathrm{O}_{2} \\
& \mathrm{BrO}_{3} \cdot h v \rightarrow \mathrm{BrO} \cdot+\mathrm{O}_{2} \\
& \mathrm{HBrO}+h v \rightarrow \mathrm{Br} \cdot+\cdot \mathrm{OH}
\end{aligned}
$$

Backward reactions that lead to the regeneration of $\mathrm{BrO}_{3}^{-}$

$$
\begin{aligned}
& \mathrm{BrO}_{2} \cdot+\mathrm{BrO}_{2} \cdot \leftrightarrows \mathrm{Br}_{2} \mathrm{O}_{4} \\
& \mathrm{Br}_{2} \mathrm{O}_{4}+\mathrm{H}_{2} \mathrm{O} \leftrightarrows \mathrm{BrO}_{2}^{-}+\mathrm{BrO}_{3}^{-}+2 \mathrm{H}^{+} \\
& 2 \mathrm{BrO}+\mathrm{H}_{2} \mathrm{O} \rightarrow \mathrm{BrO}^{-}+\mathrm{BrO}_{2}^{-}+2 \mathrm{H}^{+} \\
& 2 \mathrm{BrO}_{2}^{-}+\mathrm{H}^{+} \leftrightarrows \mathrm{BrO}_{3}^{-}+\mathrm{HBrO} \\
& \mathrm{HBrO}+\cdot \mathrm{OH} \rightarrow \mathrm{BrO} \cdot+\mathrm{H}_{2} \mathrm{O} \\
& \mathrm{HBrO}+\mathrm{Br} \cdot \rightarrow \mathrm{BrO} \cdot+\mathrm{Br}^{-}+\mathrm{H}^{+} \\
& \mathrm{Br} \cdot+\mathrm{Br}^{-} \leftrightarrows \mathrm{Br}_{2}^{-} \\
& \mathrm{Br} \cdot+\mathrm{Br} \cdot \rightarrow \mathrm{Br}_{2} \\
& \mathrm{Br}_{2}^{-}+\mathrm{Br}_{2}^{-} \rightarrow \mathrm{Br}_{3}^{-}+\mathrm{Br}^{-} \\
& \mathrm{Br}_{3}^{-} \leftrightarrows \mathrm{Br}_{2}+\mathrm{Br}^{-} \\
& \mathrm{Br}_{2}+\mathrm{H}_{2} \mathrm{O} \leftrightarrows \mathrm{HBrO}+\mathrm{H}^{+}+\mathrm{Br}^{-} \\
& \mathrm{Br}_{2}^{-}+\mathrm{HBrO} \rightarrow \mathrm{BrO} \cdot+2 \mathrm{Br}^{-}+\mathrm{H}^{+}
\end{aligned}
$$




\section{References}

1. Bard, A. J.; Parsons, R.; Jordan, J. Standard potentials in aqueous solution (Vol. 6). CRC press, 1985.

2. Chen, Z. H.; Song, X. J.; Zhang, S. J.; Wu, B. D.; Zhang, G. Y.; Pan, B. C. Acetylacetone as an efficient electron shuttle for concerted redox conversion of arsenite and nitrate in the opposite direction. Water Res. 2017, 124, 331-340.

3. Buettner, G. R. Spin trapping: ESR parameters of spin adducts. Free Radic. Biol. Med. 1987, 3 (4), 259-303.

4. Wu, B. D.; Zhang, L.; Wei, S. J.; Ou'Yang, L. Y.; Yin, R.; Zhang, S. J. Reduction of chromate with UV/diacetyl for the final effluent to be below the discharge limit. $J$. Hazard. Mater. 2020, 389, 121841.

5. Parker, K. M.; Zeng, T.; Harkness, J.; Vengosh, A.; Mitch, W. A. Enhanced formation of disinfection byproducts in shale gas wastewater-impacted drinking water supplies. Environ. Sci. Technol. 2014, 48 (19), 11161-11169.

6. Soltermann, F.; Abegglen, C.; Tschui, M.; Stahel, S.; von Gunten, U. Options and limitations for bromate control during ozonation of wastewater. Water Res. 2017, 116, 76-85.

7. Soltermann, F.; Abegglen, C.; Gotz, C.; von Gunten, U., Bromide sources and loads in swiss surface waters and their relevance for bromate formation during wastewater ozonation. Environ. Sci. Technol. 2016, 50 (18), 9825-34. 
8. Butler, R. A. Y.; Godley, A.; Lytton, L.; Cartmell, E. Bromate environmental contamination: Review of impact and possible treatment. Crit. Rev. Environ. Sci. Technol. 2005, 35 (3), 193-217.

9. Bao, M. L.; Griffini, O., Santianni, D.; Barbieri, K.; Burrini, D.; Pantani, F. Removal of bromate ion from water using granular activated carbon. Water Res. 1999, 33 (13), 2959-2970.

10. Van Hees, P. A.; U. S.; Giesler R. Low molecular weight organic acids and their Alcomplexes in soil solution-composition, distribution and seasonal variation in three podzolized soils. Geoderma. 2000, 94 (2-4), 173-200.

11. Chebbi A, C. P. Carboxylic acids in the troposphere, occurrence, sources, and sinks: A review. Atmospheric Environ. 1996, 30 (24), 4233-4249.

12. Tratnyek, P. G., Grundl, T. J., Haderlein, S. B., Eds. Aquatic Redox Chemistry, ACS Symposium Series 1071; American Chemical Society: Washington, DC, 2011.

13. Mopper K, S. W. Sources and sinks of low molecular weight organic carbonyl compounds in seawater. Mar. Chem. 1986, 19 (4), 305-321.

14. Kieber, D. J.; Stahovec, M. K. Photochemical formation of glyoxylic and pyruvic acids in seawater. Mar. Chem. 1987, 21 (2), 135-149.

15. Zhou, X. L.; Mopper, K. Photochemical production of low-molecular-weight carbonyl compounds in seawater and surface microlayer and their air-sea exchange. Mar. Chem. 1997, $56(3-4), 201-213$. 
16. Kieber, R. J.; Zhou, X. L.; Mopper, K. Formation of carbonyl compounds from UVinduced photodegradation of humic substances in natural waters: Fate of riverine carbon in the sea. Limnol. Oceanogr. 1990, 35 (7), 1503-1515.

17. Vengosh, A.; Jackson, R. B.; Warner, N.; Darrah, T. H.; Kondash, A. A critical review of the risks to water resources from unconventional shale gas development and hydraulic fracturing in the United States. Environ. Sci. Technol. 2014, 48 (15), 83348848.

18. Hem, L. J.; Efraimsen, H. Assimilable organic carbon in molecular weight fractions of natural organic matter. Water Res. 200135 (4), 1106-1110.

19. Chen, Z. H.; Li, X. C.; Zhang, S. J.; Jin, J. Y,; Song, X. J.; Wang, X. M.; Tratnyek, P. G. Overlooked role of peroxides as free radical precursors in advanced oxidation processes. Environ. Sci. Technol. 2019, 53 (4), 2054-2062. 